\title{
Modeling High Concentration Boron Diffusion with Dynamic Clustering: Influence of the Initial Conditions
}

\author{
B. Baccus and E. Vandenbossche \\ IEMN-ISEN \\ 41 Boulevard Vauban, F-59046 Lille Cédex, FRANCE
}

\begin{abstract}
Boron diffusion and activation at high concentrations are key problems in the formation of shallow $\mathrm{P}^{+}$junctions. Therefore, it is needed to understand and to predict accuratly the dopant behaviour under these conditions. In this paper, the modeling of boron is discussed, by the use of a non-equilibrium pointdefect model, including amorphization and a dynamic clustering component. The initial conditions are of major importance not only for the transient enhanced diffusion, but also for the amount of active dopant. As a result, it is possible to obtain activation levels greater than the solubility limit, as observed experimentally.
\end{abstract}

\section{The point-defect diffusion model}

A non-equilibrium point defect-model has been used throughout the study. It solves rigorously the interactions between dopants and point-defects (including charged species) and takes into account the ion implantation damage. Five coupled equations are needed to solve the diffusion of boron : substitutional boron $\mathrm{Bs}$, interstitial boron $\mathrm{Bi}$, interstitial $\mathrm{I}$, vacancy V, and Poisson equation [1]. In this frame, the simplest modeling of a boron cluster is given by : $\mathrm{Bs}+\mathrm{Bi} \leftrightarrow \mathrm{BsBi}$, where the cluster is considered as immobile and in nonequilibrium. Such cluster has been already proposed in a static form [2] or a dynamic one [3] as chosen here. This adds a sixth equation to the system. It is possible to include clusters containing more atoms, but it does not give a significant improvement.

\section{Initial conditions}

In the case of high-dose boron ion implantation, it is difficult to determine exactly the initial conditions. There is no amorphization, however it is known that extended defects can appear as well as activation levels greater than the solubility limit (Csol) [4]. The modeling 
used in the present study is a generalisation of the amorphization model proposed in [5]. In the following, the 'disordered' zone is considered as a region close to amorphization in which, (1) the boron is completely active and (2) some supersaturation of point-defects still exists (in particular interstitials). This is then an intermediate case between a relatively low-dose implant (high concentrations of $I$ and $V$ ) and the situation after solid phase epitaxy of an amorphized region. A typical example is given in fig. 1 for a $5.10^{15} \mathrm{at} / \mathrm{cm}^{2} 20$ keV boron implant.

\section{Comparison with experiments}

The calculations have been compared to several experiments including the ones from Solmi [4] and Cowern [6]. Fig. 2 shows the different species after $10 \mathrm{~s}$ of diffusion at $850^{\circ} \mathrm{C}$ (initial condition in fig. 1). The interstitial level is severely decreased in the 'disordered' zone due to the formation of the $\mathrm{Bi}$ and $\mathrm{BsBi}$ species. A high level of activation is still maintained. A good agreement between simulation and SIMS is found for different diffusion times (fig. 3) and also concerning the activation level (fig. 4). The activation is strongly dependent on the initial interstitial supersaturation (fig. 5). If the supersaturation is extremely high, all the substitutional boron will be converted rapidly to the interstitial component and the activation will be below Csol, reaching this value for long times. On the contrary, if the zone would be completely amorphized (interstitial level close to equilibrium), a very high level of activation could be obtained even for long times. As expected, the cases under study fall between these two limits, enabling activations slightly higher than Csol.

\section{Conclusion}

It has been shown that the level of activation resulting from high-concentration boron diffusion after post-implant annealing, can be controlled by the initial amount of point-defects in the 'disordered' zone. Such modeling allows to reproduce experiments for amorphizing and non-amorphizing ambients.

\section{References}

[1] B. Baccus et al., IEEE Trans. Electron Devices, ED-39, 648 (1992).

[2] D. Mathiot and J.C. Pfister, J. Appl. Phys., 55 (10), 3518 (1984).

[3] M. Hane and H. Matsumoto, IEDM Techn. Digest, 701 (1991).

[4] S. Solmi, F. Baruffaldi and R. Canteri, J. Appl. Phys., 69 (4), 2135 (1991).

[5] H. Kinoshita and D.L. Kwong, IEDM Techn. Digest, 165 (1992).

[6] N.E. B. Cowern, K.T.F Janssen and H.F.F. Jos, J. Appl. Phys., 68 (12), 6191 (1991). 


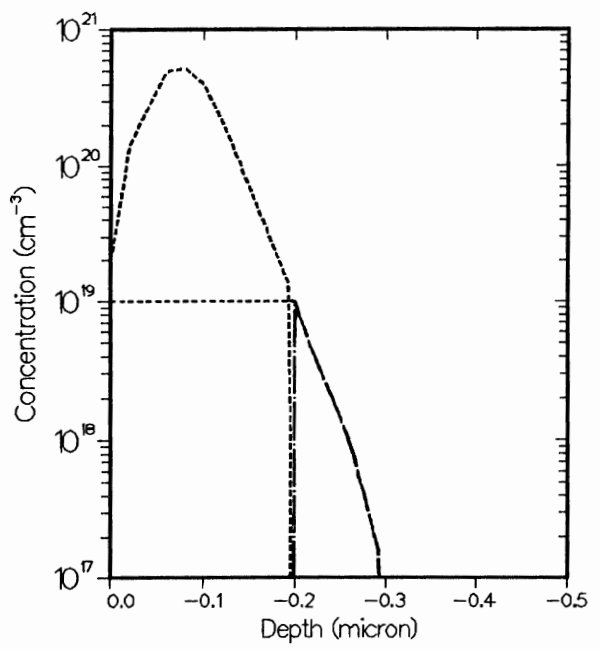

Fig. 1: Initial conditions for a $5.10^{15} \mathrm{at} / \mathrm{cm}^{2}$, $20 \mathrm{keV}$ boron implant.

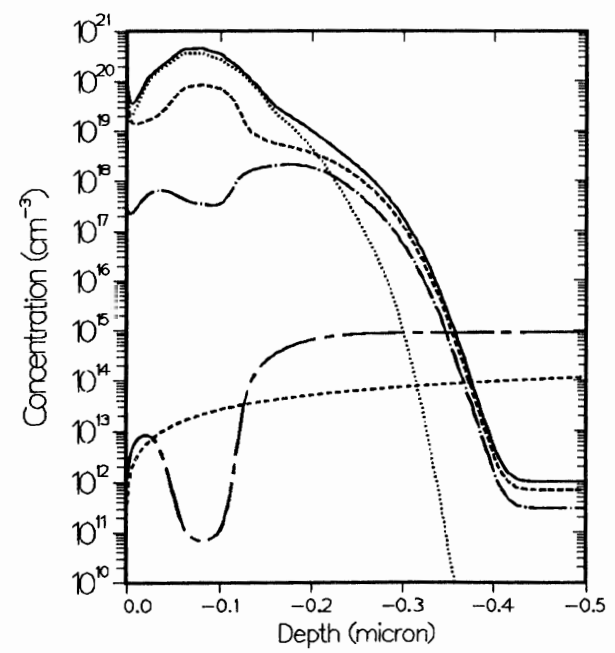

Fig. 2: Species after $10 \mathrm{~s}$ diffusion at $850^{\circ} \mathrm{C}$. 

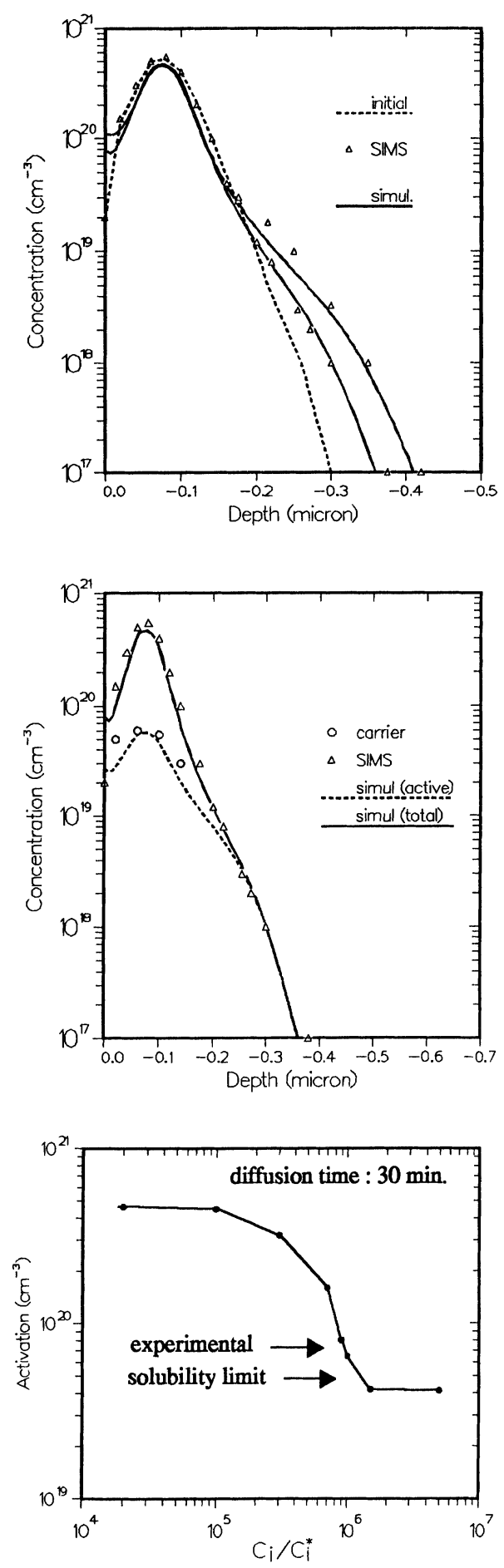

Fig. 3: Comparisons between simulation and SIMS profiles [4], after 30 $\mathrm{min}$ and $2 \mathrm{hrs}$ of diffusion at $850^{\circ} \mathrm{C}$.

Fig. 4: Comparison between simulation and experiments (SIMS and carrier profiles). After $30 \mathrm{~min}$ of diffusion at $850^{\circ} \mathrm{C}$.

Fig. 5: Evolution of the maximum active concentration as a function of the initial interstitial supersaturation. The curve is slightly shifted when varying the extent of the disordered zone. 